\title{
Fertigungsanlagen in der Urformtechnik Untersuchung des Einsatzes von automatischen kastenlosen DISAMATIC-Formanlagen
}

\author{
Norbert Miersch
}

Den Urformverfahren kommt durch die Vorteile der freien Gestaltung der Formgebung und den minimalen Werkstoffabfall im Zeitalter der steigenden Rohstoffund Energieverknappung eine immer größer werdende Bedeutung zu. In diesem Zusammenhang motiviert die ständige Weiterentwicklung und Verbreitung von automatischen Formanlagen für die produktive Herstellung von verlorenen Formen zu einer gesonderten Betrachtung einer speziellen kastenlosen Formanlage. Die Integration in den Planungs- und Gießprozess unter Berïcksichtigung der Anwendungsgrenzen der Formanlage wird im folgenden Artikel vorgestellt und untersucht.

\section{Besonderheiten und Definitionen}

Bevor auf die spezielle Fertigungsanlage eingegangen wird, sind eine Erläuterung sowie Begriffsdefinitionen notwendig. Fertigungsanlagen werden nach den Verfahrenshauptgruppen Urformen, Umformen, Trennen, Fügen, Beschichten und Stoffeigenschaftsändern gegliedert. Elemente von Fertigungsanlagen sind Werkzeugmaschinen, die diese spezielle Bezeichnung fuir die Fertigungsverfahren Umformen, Trennen und Fügen in Anspruch nehmen [12]. Eine Werkzeugmaschine ist ein Fertigungsmittel zur Erzeugung einer definierten Werkstuickform. Durch relative Bewegungen zwischen Werkzeug und Werkstück wird eine vorgegebene Form oder Veränderung am Werkstuick erreicht. Ein Werkzeug dagegen stellt dabei ein Fertigungsmittel dar, dass bei der Ausführung eines Arbeitsgangs direkt oder indirekt auf die Zustandsänderung am Werkstück oder das Füigen von Montageeinheiten wirkt. Durch eine Relativbewegung zwischen Werkzeug und Werkstück unter Energieübertragung wird eine Werkstückform gebildet oder verändert. Hiernach kann eine präzisierte Begriffsbestimmung für das Urformwerkzeug vorgenommen werden. Es ist ein auf einer Formmaschine bzw. Fertigungsanlage durch eine Maschine geführtes Wirkmittel, das durch Energieübertragung den gestaltbestimmenden und verfestigenden Zwang auf formlosen Stoff ausübt. Die zur Herstellung einmalig verwendbarer Gießformen (verlorener Formen) aus Formstoff erforderlichen Modelle und Kernkästen sind demnach Urformwerkzeuge. In der Urformtechnik sind produktive automatische Fertigungsanlagen beispielsweise Formanlagen zum Herstellen vertikal geteilter kastenloser Formblöcke. Die Erfindung dieses Formverfahrens machte die Firma Dansk Industri Syndikat A/S. Es wird deshalb weltweit unter den Namen „DISAMATIC-Formverfahren“ geführt. Die auf dieser Fertigungsanlage produzierten Formblöcke bestehen aus feuchtem, tongebundenem, Qarzsand (Grünsand) und werden zu einem Formstrang zusammengelegt. Bei der DISAMATIC-Formanlage können Modellplatten als Urformwerkzeug bezeichnet werden. Wegen der hohen Drücke, die beim Formprozess auftreten, ist es zweckmäßig, diese vorrangig aus NE-Metall, Metall oder Kunststoff herzustellen. Ein progressiver Werkzeugwechsel ist die Anwendung von Kassettensystemen. Diese werden verwendet, wenn das Produktionsprogramm aus kurzen Serien besteht bzw. Modelle anderer Formverfahren auf DISAMATIC-Modelle umgerüstet werden müissen. Die auswechselbaren Modellplatten werden dabei in standardisierte Grundplatten (Rahmen) eingewechselt und hydraulisch gespannt. Der Werkzeugwechsel wird innerhalb weniger Minuten durchgefuihrt [6].

Zur Herstellung der Gießform können auch Kerne benötigt werden. Diese werden verwendet, wenn hinterschnittene Gussteilaußenkonturen, Gussteilinnenkonturen, die einen unzulässigen Formschwierigkeitsgrad aufweisen, oder hinterschnittene Gussteilinnenkonturen nicht ausgeformt werden können. Der Formschwierigkeitsgrad wird aus dem Verhältnis von Höhe und Breite des Formballens bestimmt und sollte den Maximalwert Fs max $=2$ nicht überschreiten [1]. Zur Herstellung von Kernen existieren unterschiedliche Kernformverfahren. Ein häufig eingesetztes Kernformverfahren ist das COLD-BOX Verfahren. Ein verwendeter Kernkasten auf einer Kernschießmaschine stellt dabei das Urformwerkzeug dar.

\section{Einordnung des DISAMATIC-Formverfah- rens in die Verfahrenshauptgruppe Urformen}

In der Verfahrenshauptgruppe Urformen wird das Gießen in verlorenen Formen hauptsächlich in der Kleinund Mittelserienfertigung angewandt. Bei einer wirtschaftlichen Fertigung auf automatischen Formanlagen sollte dabei die Fertigungsstückzahl in Abhängigkeit von der jeweils betrachteten Gießerei zwischen 50 und 1000 Stück pro Jahr liegen. Dabei spielen bei der Auswahl des Form- und Gießverfahrens der Gusswerkstoff und die Massebereiche der Gussstücke eine wichtige Rolle. Die einzelnen Form- und Gießverfahren können grundsätzlich unterschieden werden nach:

- der Herstellung differenzierter Abmessungsbereiche von Gussteilen,

- der Art des Einströmens des flüssigen Metalls und damit der Art der Einformlage, 
- der Möglichkeit der Realisierung bestimmter Maßgenauigkeiten und Oberflächengüten,

- der Möglichkeit des Vergießens unterschiedlicher Gußwerkstoffe [9].

Abbildung 1 zeigt einen Auszug aus der Systematik die die Zuordnung des DISAMATIC-Formverfahrens zu der Verfahrenshauptgruppe Urformen deutlich macht.

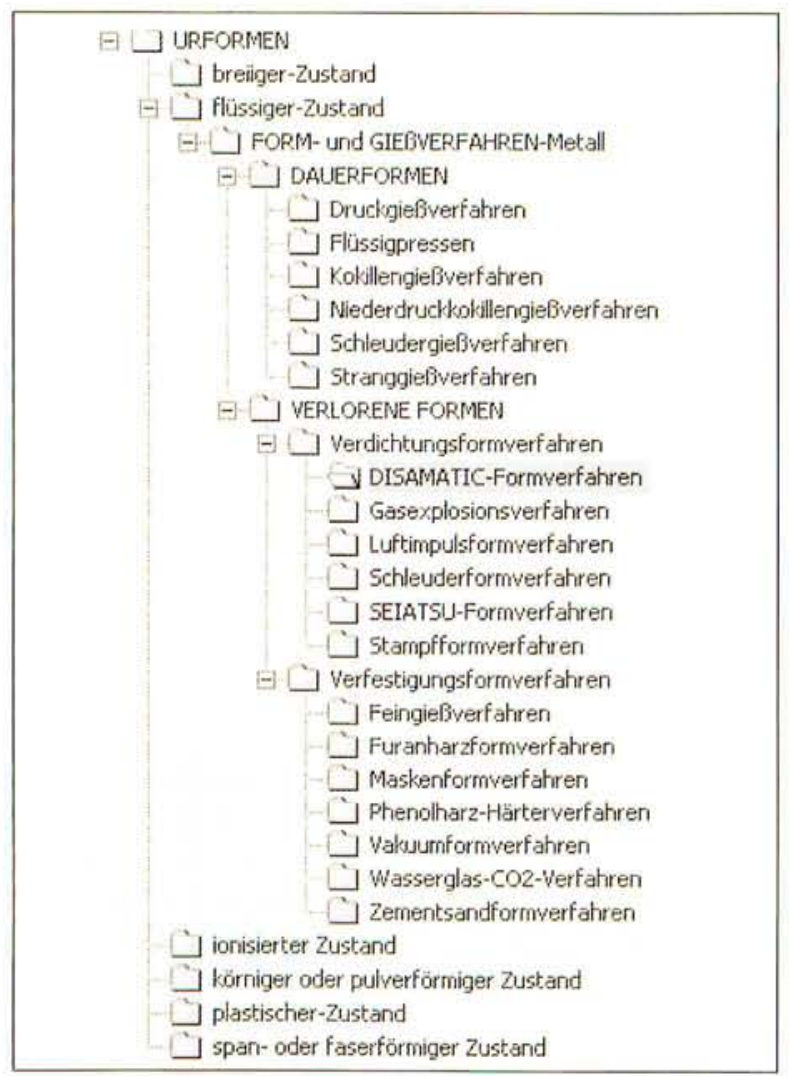

Abb. I: Einordnung des Disamatic-Formverfalurens in die Verfahrenshauptgruppe Urformen

\section{Entwicklung sowie Vor- und Nachteile des Formverfahrens}

Die Firma Dansk Industri Syndikat A/S (Disa), aus Herlev (Dänemark) stellte diese Formmaschine zum ersten Male 1962 auf der GIFA in Düsseldorf unter der Bezeichnung Disamatic vor [11]. Im Januar 1996 ging Dansk Industri Syndikat mit der Georg Fischer AG einen JointventureVertrag ein. Die Formmaschine wird jetzt offiziell von der Firma Georg Fischer DISA Holding AG vertrieben. Seit dem ersten Modell der Maschine wurde sie bis heute kontinuierlich weiterentwickelt. In Abhängigkeit vom Formenvorschub können mit der neuen DISAMATIC-Generation kernlos bis zu 500 Formen pro Stunde und mit dem Einlegen von Kernen bis zu 400 Formen pro Stunde geformt werden. Die Implementierung von modernen PLC-Programmen optimiert sämtliche Funktionen und Bewegungen des mechanischen und hydraulischen Systems. Es wird ein maschinenbedingter Versatz von $0,1 \mathrm{~mm}$ garantiert.

Nach Recherchen in |4| und einer nachfolgenden Auswertung kann in 350 nachgeprüften Gießereien des Deutschen Gießereiverbandes festgestellt werden, dass 270 Gießereien Maschinenformanlagen besitzen und 110 Gießereien mit Maschinenformanlagen DISAMATIC-
Formanlagen besitzen (siehe Abb. 2). Disamatic Formanlagen sind weltweit in über 60 Ländern im Einsatz [7].

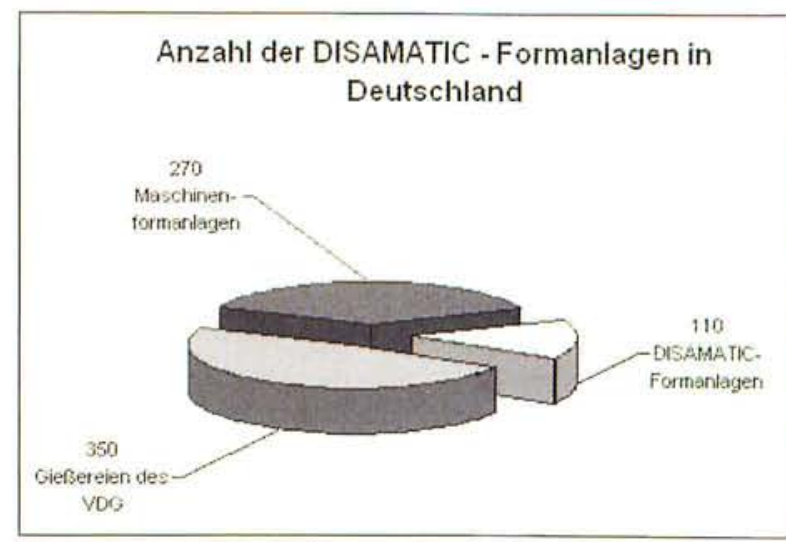

Abb. 2: Anteil der DISAMATIC-Formanlagen in Deutschlands Gießerein

Bei einem Vergleich des DISAMATIC-Formverfahrens mit produktiven horizontalen kastengebundenen Formverfahren weist das Verfahren folgende Vorteile auf:

1. Es entfallen Kosten für die Bindung von Formkästen (Oberkasten/Unterkasten) an das herzustellende Gussteil.

2. Durch das Gießen im Formstrang entfallen zusätzliche Kastenbeschwerungen oder Kastenverklammerungen, um den Auftriebskräften beim Gießen entgegenzuwirken.

3. Hohe Produktivität der Anlage. Angaben in |5| mit 400 bis 500 Formen/Stunde in Abhängigkeit von einzulegenden Kernen.

4. Es ist nur ein Maschinenbediener (Former) notwendig, der auch während des Formprozesses Kerne mit Hilfe eines Kerneinlegers in den Formhohlraum einlegen kann.

5. Hohe Maßgenauigkeit beziiglich Formversatz von $0,1 \mathrm{~mm}$.

6. Gleichmäßig hohe Formhärte.

7. Für die Formanlage wird kein spezielles Fundament benötigt.

Nachteile des Formverfahrens sind:

1. Einschränkung des Teilesortiments durch relativ geringe Formblockabmessungen.

2. Beachtung von Restriktionen bezüglich der gesonderten Kernhandhabung durch Kerneinleger.

3. Besondere Forderungen an die Kernlager und Kerngeometrie.

4. Höhere Anforderungen an den Modellwerkstoff (Holz ist nicht zweckmäßig).

5. Beachtung von Restriktionen beziiglich der vertikalen Formteilung.

\section{Aufbau und Funktion der DISAMATIC-Formanlage}

\subsection{Aufbau}

Die DISAMATIC - Formanlage besteht aus verschiedenen Funktionsbaugruppen (siehe Abb. 3), wobei die wichtigsten im Weiteren näher erläutert werden. 


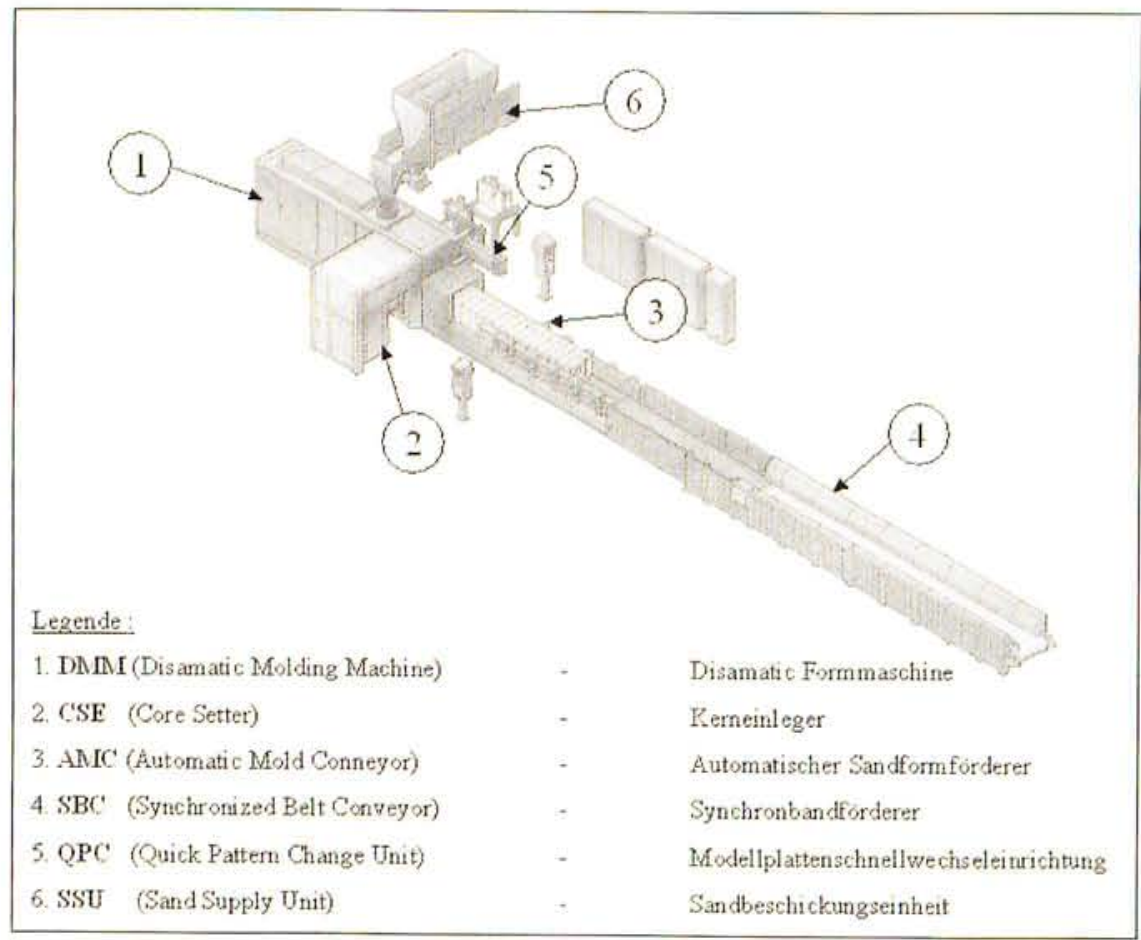

Abb. 3: Aufbau der DISAMATIC-Formanlage

\subsection{Die Funktionsweise der Formmaschine (DMM)}

Der Herstellungsablauf der Formblöcke wird in Bezug auf das Wirkprinzip der Formmaschine als Arbeitsstufe bezeichnet (siehe Abb. 4). Eine Arbeitsstufe ist ein Abschnitt des Arbeitsgangs ohne Veränderung der Arbeitsgegenstandslage [10]. Zur Herstellung eines Formblokks sind sechs Arbeitsstufen notwendig, die im Folgenden für eine DISAMATIC 2110 MK3 mit PLC-Steuerung (PLC oder SPS sind speicherprogrammierbare Steuerungen. Sie ersetzen in ihrer einfachsten Ausführung frühere Relaissteuerungen.) kurz erläutert werden.
Arbeitsstufe 1: Sand wird in die Formkammer mit einem Druck von ca. 3 bar eingeschossen. Der einstellbare Sandschussdruck kann zwischen 0 bis 5,5 bar verändert werden. Die Formkammer und die Sandschleuse sind während des Schießens geschlossen. Durch Öffnen des Sandschussventils wird die Formkammer mit Formsand gefüllt. In der PLC-Steuerung kann dabei noch eine Korrektur der Sandschusszeit von $+/-0,5 \mathrm{~s}$ erfolgen.

Arbeitsstufe 2: Die Schwingplatte und die Pressplatte drükken den in der Formkammer befindlichen Formsand soweit zusammen, bis der in der PLC eingestellte Pressdruck erreicht ist. Der Pressdruck der beiden sich aufeinander zubewegenden Modellplatten wird im Normalfall auf $8 \mathrm{Kp} / \mathrm{cm}^{2}$ eingestellt. Er kann aber zwischen 4 und $12,5 \mathrm{kp} / \mathrm{cm}^{2}$ variiert werden. Die Presszeit kann bei Bedarf um $0,5 \mathrm{~s}$ verlängert werden. Zwischen den beiden Modellplatten kann weiterhin eine Pressverzögerung eingestellt werden. Nach dem Preßvorgang wird die Sandschleuse wieder geöffnet und der Sandbehälter kann nachbeschickt werden.

Arbeitsstufe 3: Abziehen der Schwingplatte von der Form. Das Abheben kann mittels Einstellung in der PLC durch eine Vibration unterstützt werden. Dabei bleiben die Pressplatte und der Formblock immer in gleicher Positi-
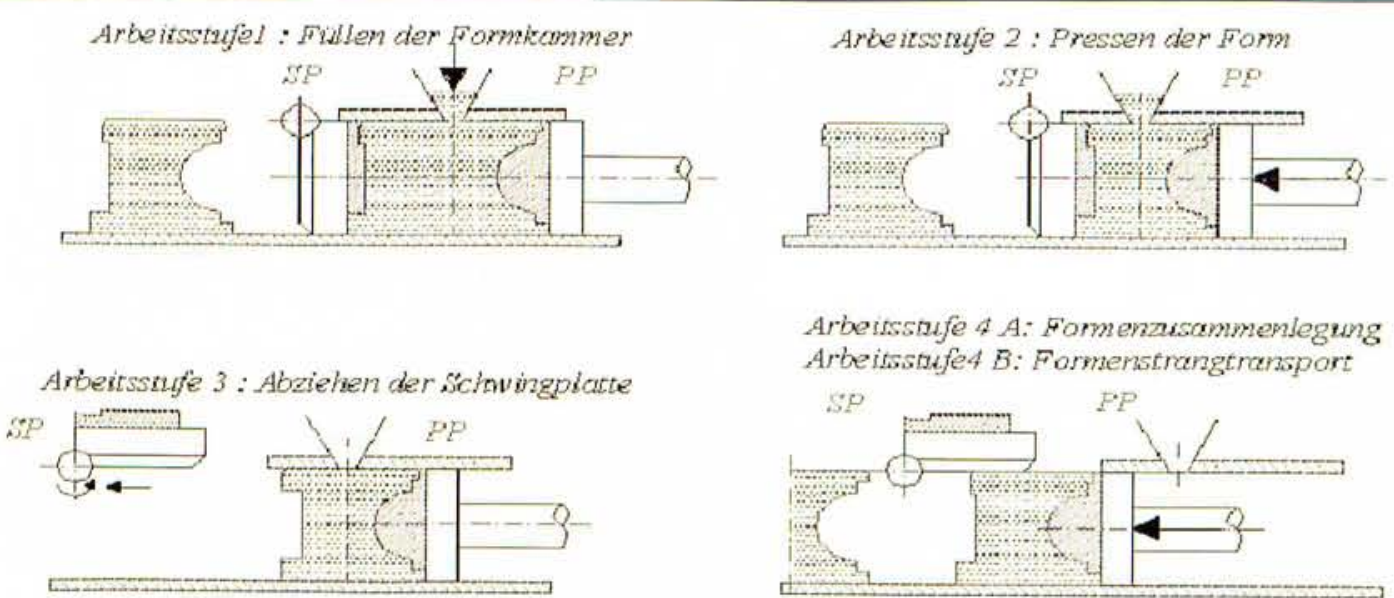

Arbeitssife \& A: Fom enzusammenlegung Arbeitssufe4 B: Formenstrangtransport

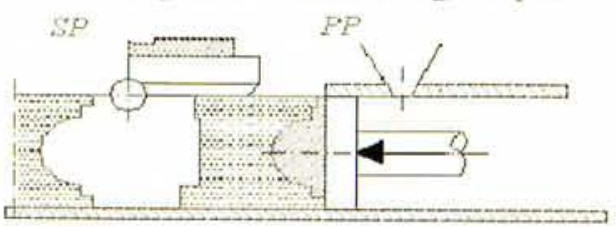

Arbeitsstufe 5: Abziehen der Preßplatte

Arbeitsstufe 6: Schließen der Fornkammer
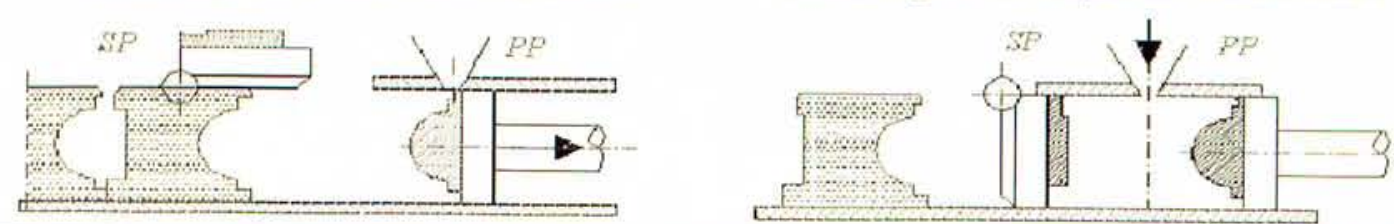

Abb. 4: Formarbeitsgang der DISAMATIC-Formmaschine $(S P=$ Schwingplatte, $P P=$ Preßplatte) 
on zueinander (Vermeidung von Formungenauigkeiten). Durch eine optionale Beheizung der Schwingplatte kann der Entformungsvorgang erleichtert werden. Nach dem Abziehen dreht sich die Schwingplatte in eine horizontale Position. Eine Abhebebeschleunigung zwischen 0,2 und $1 \mathrm{~m} / \mathrm{s}^{2} \mathrm{kann}$ in der PLC eingestellt werden. Der Standardwert ist dabei $0,5 \mathrm{~m} / \mathrm{s}^{2}$. Die Formkammer ist jetzt offen.

Arbeitsstufe 4 a): Die Pressplatte schiebt die Form aus der Formkammer der Maschine zum Formablieferungspunkt. Die Zusammenlegungsposition muss korrekt eingestellt und in die PLC-Steuerung eingetragen werden. Der Formzusammenlegungsdruck kann zwischen 0 und 1 bar variieren, wobei der Standardwert bei 0,7 bar eingestellt wird. Während der Übergabe des Formblocks an den Formstrang muss die Pressplatte abgebremst werden. Dieser Pressplatten-Abbremsdruck kann zwischen 0 und 4 bar eingestellt werden. Der Standardeinstellwert liegt bei 1 bar.

Arbeitsstufe 4 b): Nach der Übergabe der Form durch die Pressplatte an den Formstrang am Formablieferungspunkt übernimmt der automatische Sandformförderer den Formblock und schiebt ihn mit dem gesamten Formstrang um eine Form weiter. Die Pressplatte darf während der Übergabe einen vorgegebenen Druck nicht überschreiten. Dieser liegt bei 1 bar und kann zwischen 0 und 4 bar variieren.

Arbeitsstufe 5: Die Pressplatte wird jetzt abgehoben, dabei begünstigt eine einstellbare Modellplattenerwärmung die Entformung. Eine Vibration kann auch eingestellt werden. Die Pressplatte fährt mit einer standardmäßigen Beschleunigung von $0,5 \mathrm{~m} / \mathrm{s}^{2}$ in die Ausgangsposition. Die Beschleunigung der Pressplatte (PP) kann in einem Intervall zwischen 0,2 bis $1 \mathrm{~m} / \mathrm{s}^{2}$ verändert werden.

Arbeitsstufe 6: Die Schwenkplatte schwingt in die vertikale Stellung zurüick und schließt die Formkammer für einen neuen Zyklus.

\subsection{Beschreibung wichtiger Baugruppen}

\section{Der Kerneinleger (CSE)}

Der Kerneinleger arbeitet, während die Formkammer geschlossen ist, vom Ende der Arbeitsstufe sechs bis zu Beginn der Arbeitsstufe drei. Er legt Kerne in die zuletzt hergestellte Form ein, während die Disamatic-Formmaschine eine neue Form produziert. Die Kerne werden durch den Maschinenbediener möglichst in der Zeit während der Arbeitsstufe drei und Arbeitsstufe sechs in eine Kerneinlegermaske eingelegt. Die Kerneinlegermaske dient zur Platzierung der Kerne in den Formhohlraum des Formblocks der durch die Pressplatte geformt wurde. Grundsätzlich stellt die Kernmaske den negativen Abdruck des Schwingplattenmodells dar. Die Bestückung des Formblockes mit den notwendigen Kernen erfolgt stufenweise und kann folgendermaßen beschrieben werden:

1. Der Bediener setzt während der Arbeitsstufe drei bis zur Arbeitsstufe sechs die Kerne in die Kerneinleger- maske des Kerneinlegers ein. Dabei wird/werden der Kern/die Kerne in der Kerneinlegermaske durch einen Unterdruck im Kernlager der Maske festgehalten. Der Unterdruck wird durch eine Vakuumpumpe an der Maschine erzeugt. Dieser beträgt bei der DISAMATIC 2110 MK3-Formmaschine 0,18 bar. Die Ausführung der Kernlager wird in [6] ausfuihrlich beschrieben. Beim Kerneinlegen ist die Kerneinlegetür geöffnet.

2. Nach der Bestïckung der Kerneinlegermaske durch den Bediener wird die Kerneinlegertiir geschlossen. Ist die Formkammer geschlossen, schwingt der Kerneinleger mit Kermmaske in Stellung.

3. Der Kerneinleger bewegt sich auf den Formblock zu. Dieser übergibt den Kern/die Kerne an den Formblock. Der Kern/die Kerne müssen durch eine spezielle Kernlagerung, die von der Form und der Masse der Kerne abhängt, im Formblock beim Zurüickziehen der Kerneinlegermaske hängen bleiben. Die Ausführung der Kernlagerung wird in [6] ausfuihrlich beschrieben.

4. Der Kerneinleger mit Kernmaske bewegt sich vom Formstrang weg.

5. Der Kerneinleger mit Kernmaske schwenkt in die Ausgangsposition zuriick.

Die Formmaschine und der Kerneinleger sind synchronisiert, d. h. es erfolgt eine zeitliche Abstimmung der beiden Funktionseinheiten, die die Taktzeit der Maschine beeinflusst.

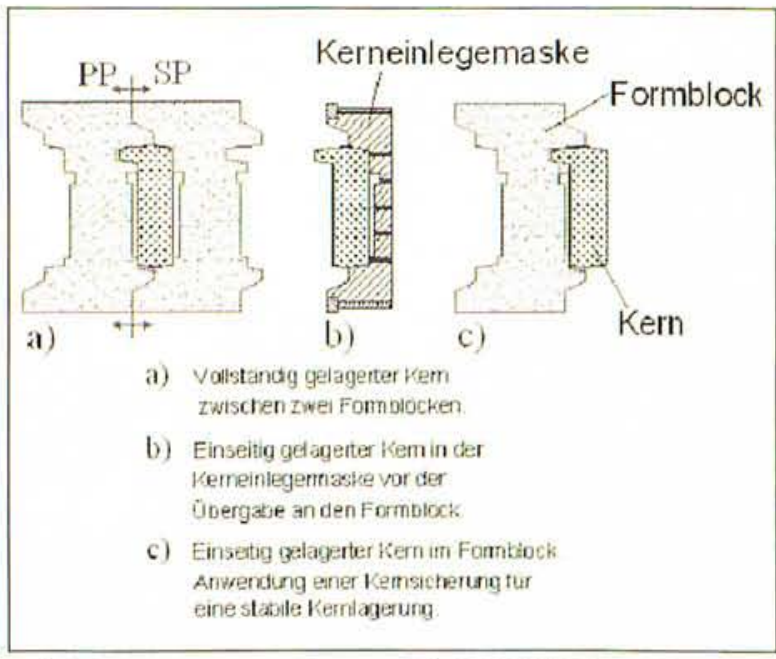

Abb. 5: Lagerungsarten von Kernen auf der DISAMATIC-Formanlage

\section{Der automatische Sandformförderer (AMC)}

Der automatische Sandformförderer bewegt den Formstrang durch die Gieß-, Erstarrungs- und Kuihlzonen. Der automatische Sandformförderer ist ein Gießrost mit schwimmend gelagerten pneumatischen Klemmschienen. Der Arbeitszyklus besteht aus vier Stufen, die in Abbildung 6 dargestellt sind. Die kontrollierte Synchronisation der Bewegungen der Formmaschine und des automatischen Sandformförderers gewährleisten eine optimale Kontrolle der Kräfte zwischen den Formen [5].

\section{Der Synchronbandförderer (SBC)}

Der Synchronbandförderer verlängert die Kühlstrecke des automatischen Sandformförderers (siehe Abb. 4). Diese Baugruppe ist notwendig, wenn eine längere Kuihlzeit der vergossenen Formen benötigt wird, ohne dass 

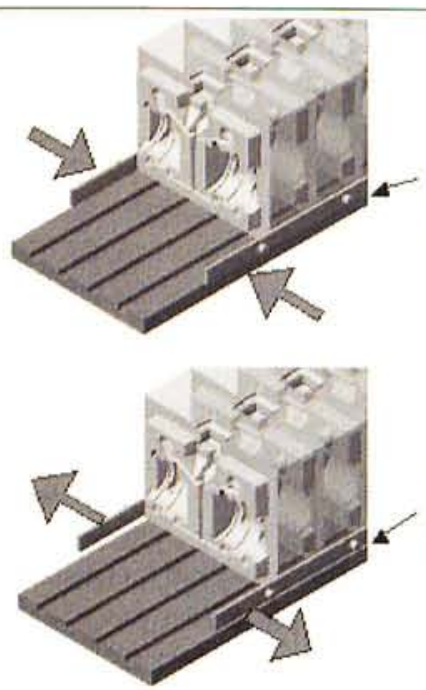

Stufe 3 :

Abheben der

AMC-Schienen.

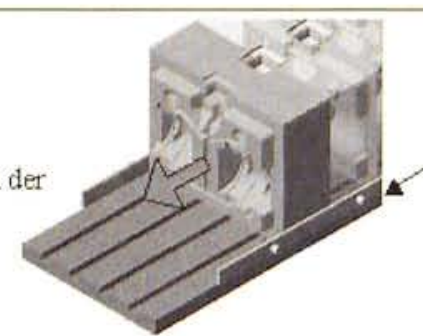

Stufe ?

Formtransport sychron mit der Presplatte in

Arbeitsstufe 4 b)

Vorschub:

Eine Form pro Arbeitstakt.

Abb. 6: Arbeitsweise des automatischen Sandformförderers

der Formprozess durch Anhalten der Formmaschine um eine bestimmte Kühlzeit unterbrochen werden muss. Der Synchronbandförderer ist mit der pneumatischen Klemmkupplung des automatischen Sandformförderers mechanisch verbunden und synchronisiert. Das Gummiband ist hitzebeständig. Der Synchronbandförderer arbeitet wie der automatische Sandformförderer in vier Arbeitsstufen (siehe Abb. 7).

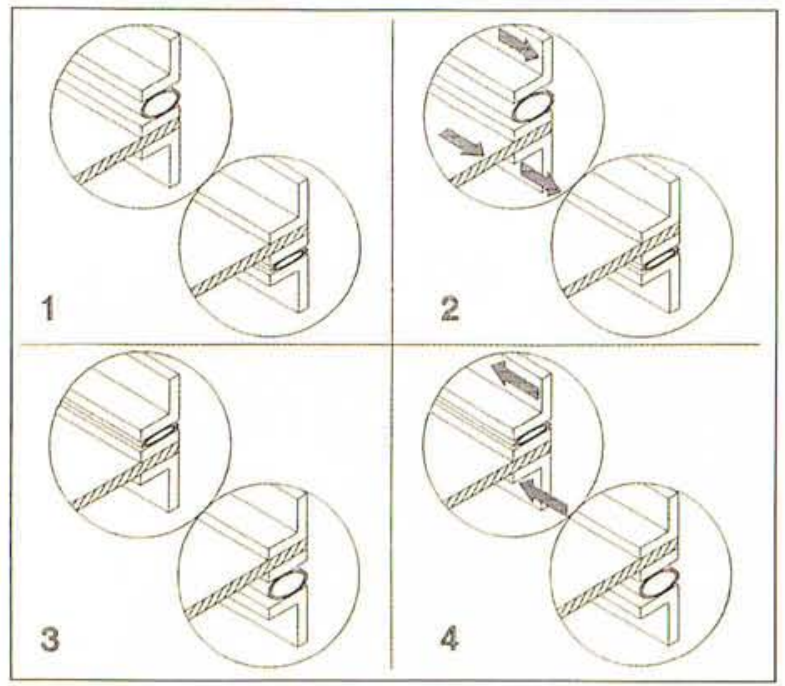

Abb. 7: Arbeitsweise der Kupplung des Synchronbandförderers

Stufe 1: Klemmkupplung mittels Druckluft aktivieren.

Stufe 2: Synchron mit dem automatischen Bandförderer wird der Formstrang um eine Form weiter bewegt.

Stufe 3: Ablassen der Luft aus der Klemmkupplung.

Stufe 4: Zurückkehren der Klemmkupplung in die Ausgangsposition.

\section{Die speicherprogrammierbare Steuerung (PLC)}

Die Disamatic-Formanlagen der neuen Generation sind mit einer modernen PLC-Steuerung ausgeruistet. Im Basisprogramm der Steuerung können alle modellspezifischen Eingabedaten verwaltet werden (siehe 4.2 Funktionsweise der Formmaschine). Die Steuerung macht eine Positions- und Geschwindigkeitsuiberwachung aller beweglichen Bauteile möglich. Sie steuert die
Synchronisation der Hauptbaugruppen. Weiterhin kann die Verdichtbarkeit der Sandform permanent überprüft werden und eine Selbstdiagnose der elektrischen Hardware zur raschen Fehlerbeseitigung erfolgen.

\section{Berücksichtigung des Einsatzes der Formmaschine in der Fertigungsplanung}

\subsection{Aufgabe der Fertigungsvorbereitung}

Der Fertigungsvorbereitung (Arbeitsvorbereitung) kommt in einem Unternehmen eine wichtige Rolle zu. Neben der Konstruktion entscheidet sie im Wesentlichen über die Höhe der Kosten eines Bauteils. [9] Die Fertigungsvorbereitung wird in die Teilbereiche Fertigungsplanung und Fertigungssteuerung untergliedert (siehe Abb. 8). WAS, WIE und WOMIT sind die zu lösenden Kernaufgaben der Fertigungsplanung. Diese gliedert sich in eine Grob- und eine Feinplanung, wobei für eine Variantenbewertung die Feinplanung die notwendigen Informationen liefert. Dabei beziehen sich beim Urformen die eigentlichen fertigungsvorbereitenden Aufgaben auf das Rohteil.

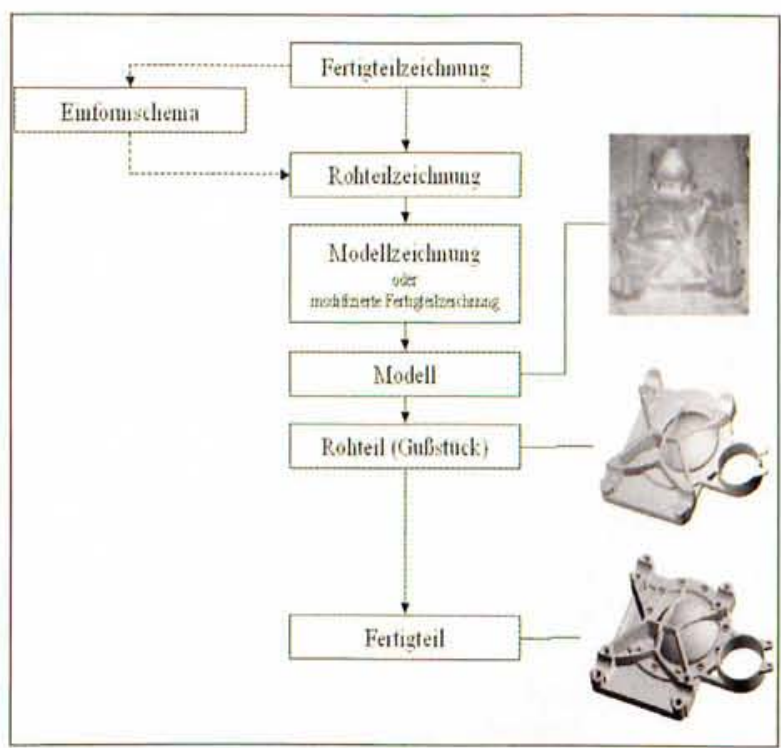

Abb. 9: Ablauf der Fertigung von Einzelteilen 


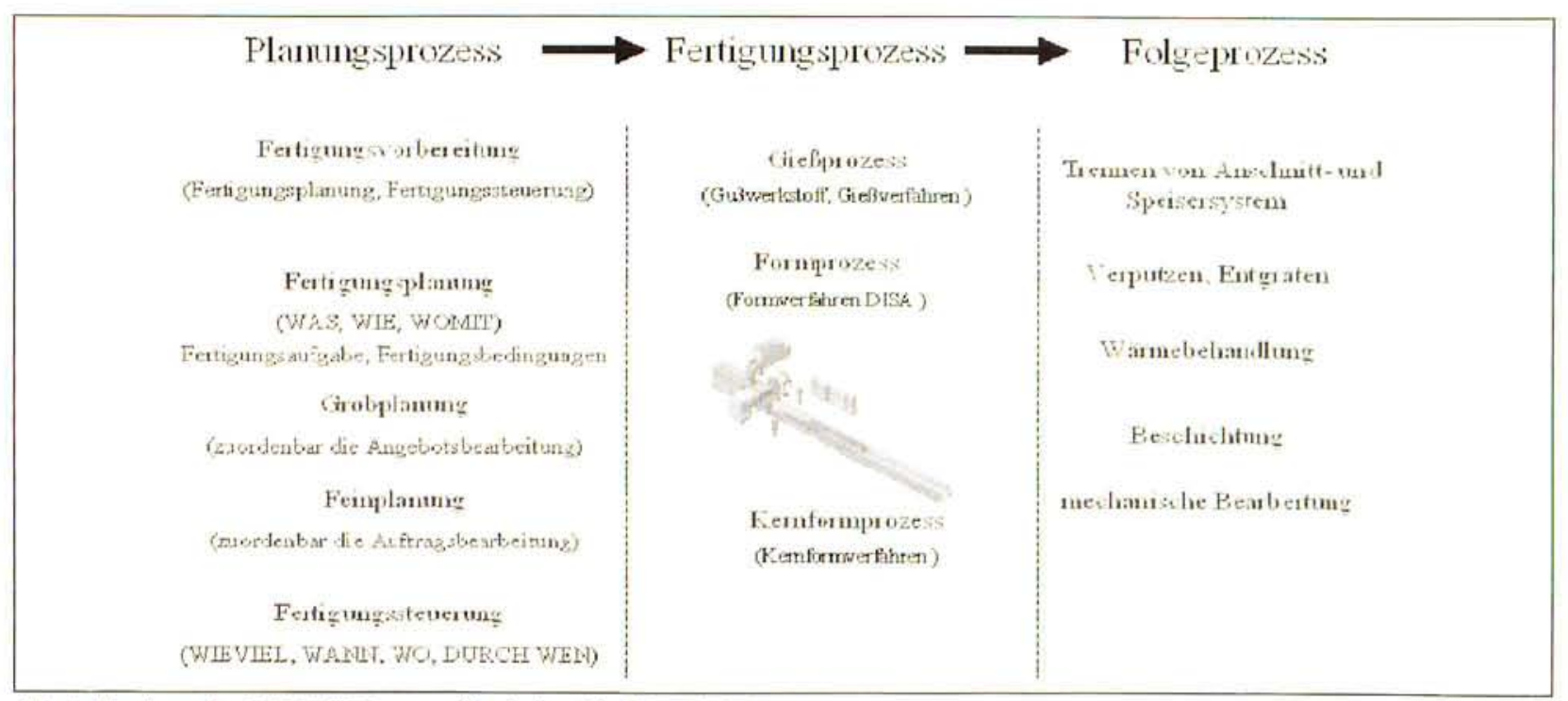

Abb. 8: Einordnung der DISAMATIC-Formmaschine in den Ablauf der Teilefertigung

\subsection{Die Einordnung der Urformwerkzeug- herstellung in den Ablauf der Fertigung}

Der Ablauf der Fertigung von Einzelteilen bezogen auf die Wahl des Rohteiles, das durch Urformen (Gießen) hergestellt wird, ist in Abbildung 9 dargestellt. Die Voraussetzung zur Fertigung eines Modells ist die Modellzeichnung. Diese stellt eine Ergänzung der Rohteilzeichnung um gießtechnologische Angaben dar [9]. Die Modellzeichnung wird oft vom Modellbauer selbst angefertigt. Eine Rohteilzeichnung verkörpert das Gussstiick nach der Entnahme aus der Form und dem Putzen ohne Kernmarken und ohne Anschnitt- und Speisersystem [9]. In der Klein- und Mittelserienfertigung ist es üblich, dass die Kundengießerei die Rohteilzeichnung nach den Erfordernissen und Besonderheiten der eigenen Fertigung selbst erstellt. In Abhängigkeit vom Auftragsvolumen und der Austattung der jeweiligen Gießerei wird oft nur eine modifizierte Fertigteilzeichnung angefertigt.

\subsection{Einordnung maschinenspezifischer Restriktionen in die Rohteilgenerierung}

Die Hauptphasen der Arbeitsvorbereitung in Unternehmen mit auftragsgebundener Fertigung sind die Angebots- und Auftragsbearbeitung. Bereits in der Angebotsphase (Grobplanung) ist es notwendig, Informationen zu vorhandenen Parametern der Ausrüstungen zu haben. Dadurch kann die technische Realisierbarkeit eines angefragten Gussteils beurteilt werden. In der Auftragsphase (Feinplanung), in Einzelfällen auch in der Angebotsphase, sind detailliertere Kenntnisse über die Anwendungsgrenzen (Restriktionen) der DISAMATIC-Formanlage und den Besonderheiten des Formverfahrens in Bezug auf das zu fertigende Rohteil notwendig.
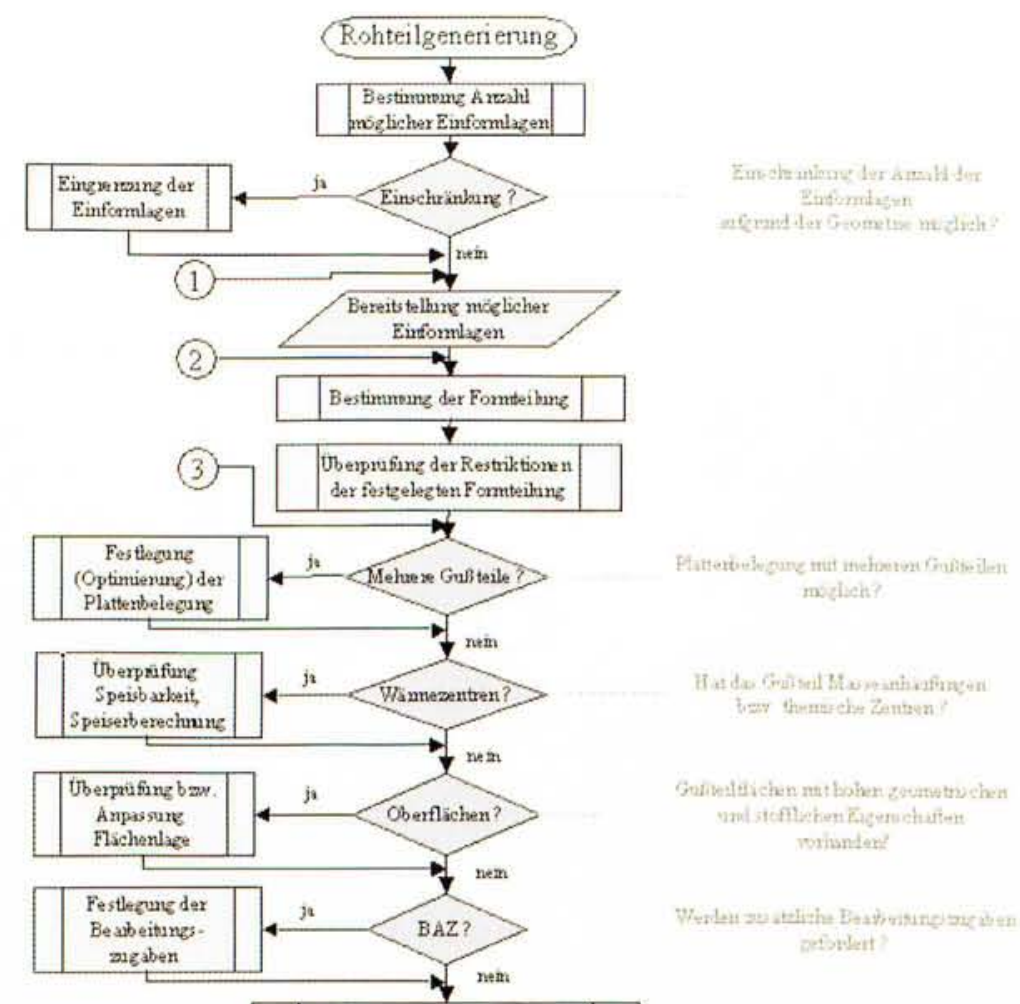

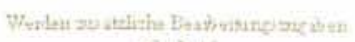
ontistert?

Bestimuning der Aushebeschrizen

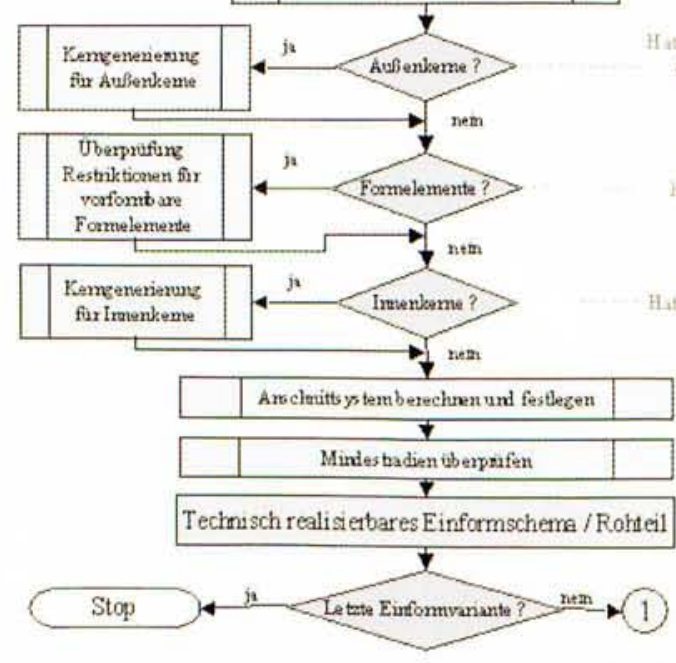

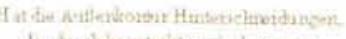

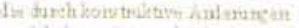

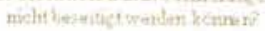

Toumon Forimtements witzaral

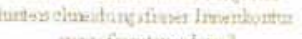
varefornt warean?

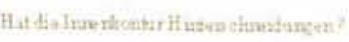

Abb. 10: Datenflussplan zur Generierung technisch realisierbarer Rohteilvarianten auf einer DISAMATIC-Formanlage 
Die Festlegung der Rohteilparameter kann nach folgenden Ablauf in Abbildung 10 durchgefuihrt werden. Im Ergebnis dieses Ablaufs können mehrere Varianten entstehen. Es fehlt dann noch eine anschließende Wirtschaftlichkeitsbetrachtung [2].

Die Überprüfung der Restriktionen der Formanlage beziiglich der Formteilung wird im Folgenden näher betrachtet. Die Festlegung der Einformlage und der Formteilung sind bereits am Rohteil notwendig und Voraussetzung zur Überprüfung der Restriktionen, die die Abmessungen des Formblocks und der Formkammer einer DISAMATIC betreffen.

\subsection{Die Einformlage als Ausgangsparameter}

Die Einformlage ist die Basis zur Generierung des Rohteiles. Sie berüicksichtigt die Lage des Gussteils im Formkasten oder im Formblock. Nach [3] wird die Einformlage als Lage oder Richtung bezeichnet, in der das Modell eingeformt bzw. aus der Form gezogen wird. Zur Betrachtung der Definition von grundsätzlichen Einformlagen eines Gussteils bezogen auf das DISAMATIC-Formverfahren werden folgende Konventionen getroffen:

1. Um das Gussteil wird ein Hüllkörper gelegt. Der Hüllkörper vereinfacht die Betrachtungen zur Einformlage, insbesondere bei komplizierten Gußteilgeometrien.

2. Es wird ein Formblockkoordinatensystem definiert, da zur Festlegung der Einformlage die Einformungsrichtung von Bedeutung ist. Die Achsen werden mit $\mathrm{u}, \mathrm{v}, \mathrm{w}$ bezeichnet.

3. Es wird ein Gußteilkoordinatensystem $x, y, z$ definiert, welches man sich fix am Rand der Hüllkontur vorstellen kann (siehe Abb. 11). Dieses Koordinatensystem wird als lokales Gussteilkoordinatensystem bezeichnet. Mit der Generierung der Einformlagen verändert es seine Beziehung zum Formblockkoordinatensystem.

\section{Festlegung von Einformlagen}

These: Im dreidimensionalen Raum gibt es sechs grundlegende Einformlagen. Diese können aufgrund symmetrischer Teilegeometrie eingegrenzt (vermindert) werden.

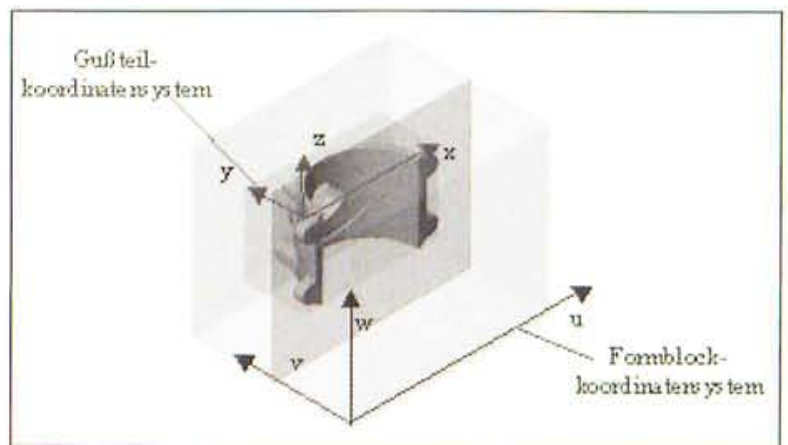

Abb. 11: Referenzen zur Bestimmung möglicher Einformlagen

Beweis:

1. Festlegung der Grundposition (Initialisierung). Wenn das Gussteil wesentliche Dimensionsunterschiede in den Hauptachsen aufweist, sollte es mit seiner größten Ausdehung (Dimension) parallel zur Gussteil-x-Achse ausgerichtet werden. Die Gussteilachsen $x, y, z$ sollten parallel $z u$ den den Achsen $u, v, w$ des Formblockes liegen. (Koordinatensystemtranslation)

2. Die Drehung (Rotation) des Gussteils in $90^{\circ}$-Winkelschritten um die w-Achse des Formblockkoordinatensystems erzeugt vier grundsätzliche Einformlagen im Formblock (siehe Abb. 12). Die Ausrichtung der Einformlage erfolgt durch eine Ausrichtung einer Achse des Gussteilkoodinatensystems ( $x$ oder y) parallel zu einer Achse des globalen Koordinatensystems (u oder v).

3. Zwei weitere Einformlagen können durch die Drehung des Gussteils von $90^{\circ} \mathrm{um}$ die u-Achse des Formblockkoordinatensystems generiert werden. Dabei ist die Einformlage von der Drehrichtung um die u-Achse von Bedeutung (positiver oder negativer Drehsinn). Abbildung 13 stellt weitere zwei mögliche Einformlagen am Beispiel einer Halbschale dar. Dabei zeigt Abbildung 13 a) die Initialisierungsansicht, Abbildung 13 b) eine weitere Einformlage und Abbildung $13 \mathrm{c}$ ) und $13 \mathrm{~d}$ ) die andere Einformlage. Dabei ist die Drehung um die v-Achse für die Definition nicht von Bedeutung.

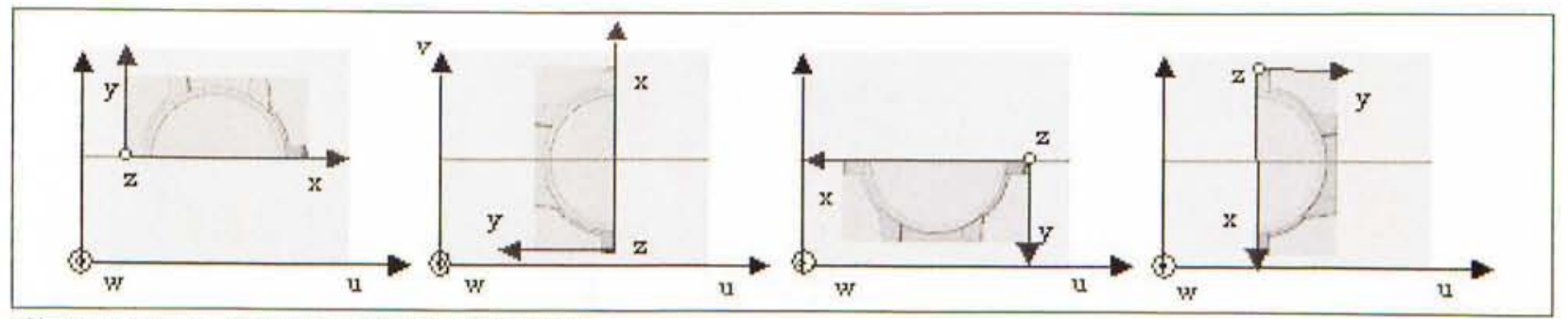

Abb. 12: Generierung von vier grundsätzlichen Einformlagen

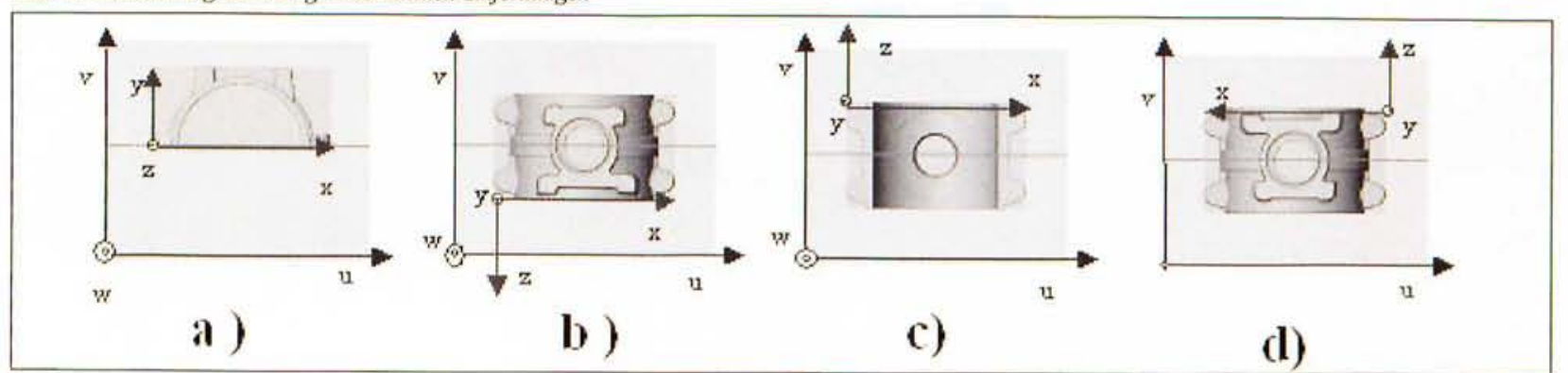

Abb. 13: Generierung zweier weiterer Linformlagen 


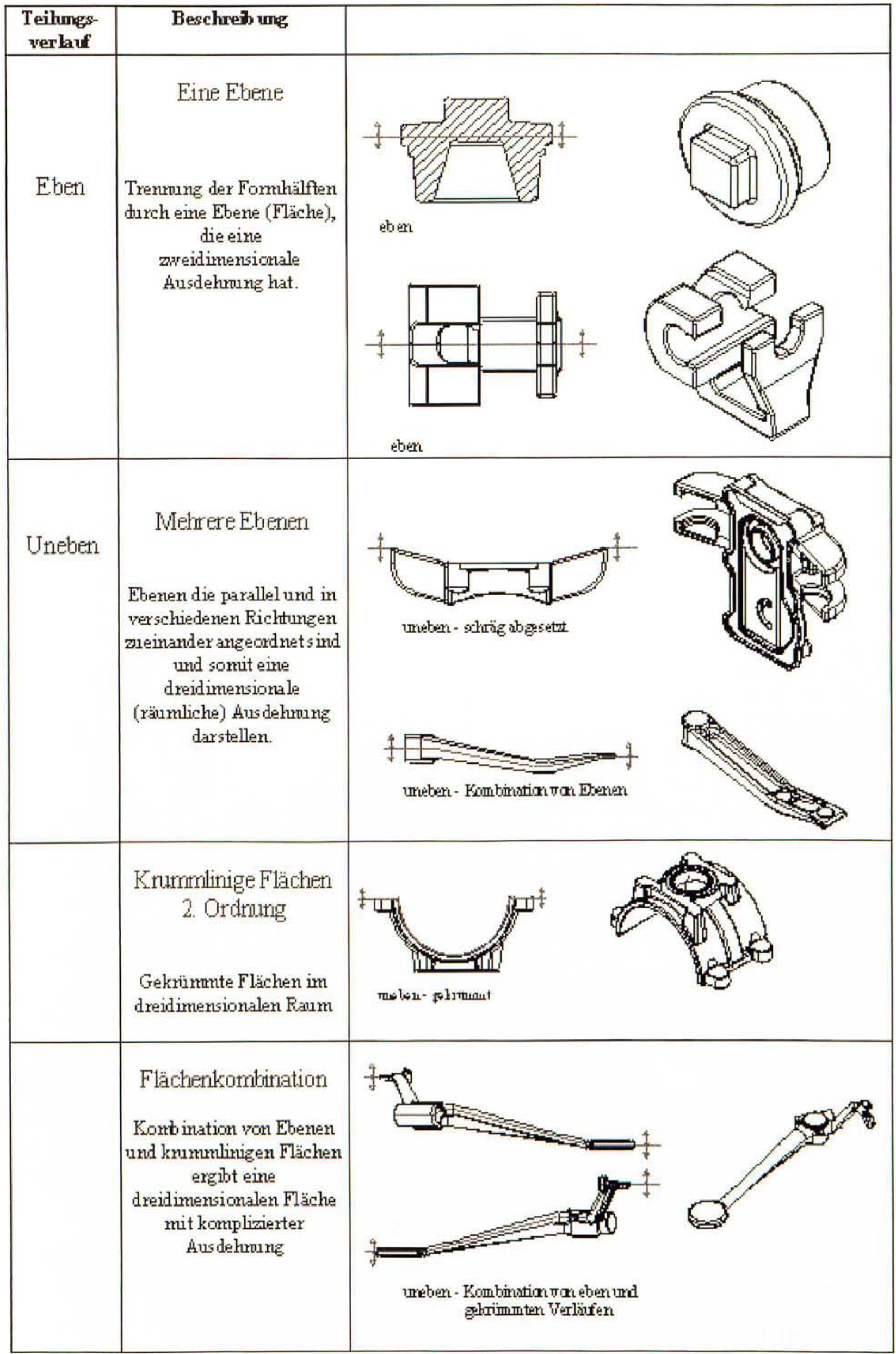


4. Liegt bezuiglich des Gussteils in einer Ebene Symmetrie vor, so vermindert sich die Anzahl der Einformlagen um die Anzahl der Symmetrieebenen. Ein rotationssymetrisches Gussteil, dass auch senkrecht zur Rotationsachse eine Symmetrie aufweist, hat dann nur zwei Einformlagen.

\subsection{Die Formteilung}

Die Teilung stellt die Trennfläche einer geteilten Gießform dar [3]. Die Entfernung des Dauermodells (Urformwerkzeug) aus der verlorenen Form, sowie der Gußstuicke aus der Dauerform setzt eine Teilung voraus. Die Teilung kann bezogen auf das DISAMATIC-Formverfahren als Kontaktfläche zwischen zwei Formblöcken definiert werden. Die Formblöcke sind vertikal geteilt. Es liegt hier eine Einfachteilung vor. Teilungen können verschiedene Teilungsverläufe aufweisen. Abbildung 14 systematisiert angewandte Teilungsverläufe für das DISAMATIC-Formverfahren.

\section{Berechnungsgrundlagen zur Überprüfung ausgewählter Restriktionen}

\subsection{Berechnung der Kammertiefe}

Die Kammertiefe der Formanlage ist durch die Entfernung der beiden Heizplatten der Formkammer vor dem Pressen definiert |6|. Die Heizplatten sind unmittelbar mit den Modellplatten verbunden. Die Überprüfung der Restriktionen bezogen auf den komprimierten Formblock berüickichtigen nicht die genaue Realisierbarkeit eines angefragten bzw. zu fertigenden Gussteiles. Die Überpriffung der Kammertiefe der Formanlage ist im Unterprogramm „Überprüfung der Restriktionen der festgelegten Formteilung" enthalten (siehe Abb. 10 und Abb. 15). Der Ablauf der Berechnung wird im Folgenden beschrieben.

Zur Berechnung der Kammertiefe sind folgende Festlegungen und Überprüfungen vorzunehmen:

A Vermeidung des Zusammenstoßens der Modellplatten nach dem Formprozess.

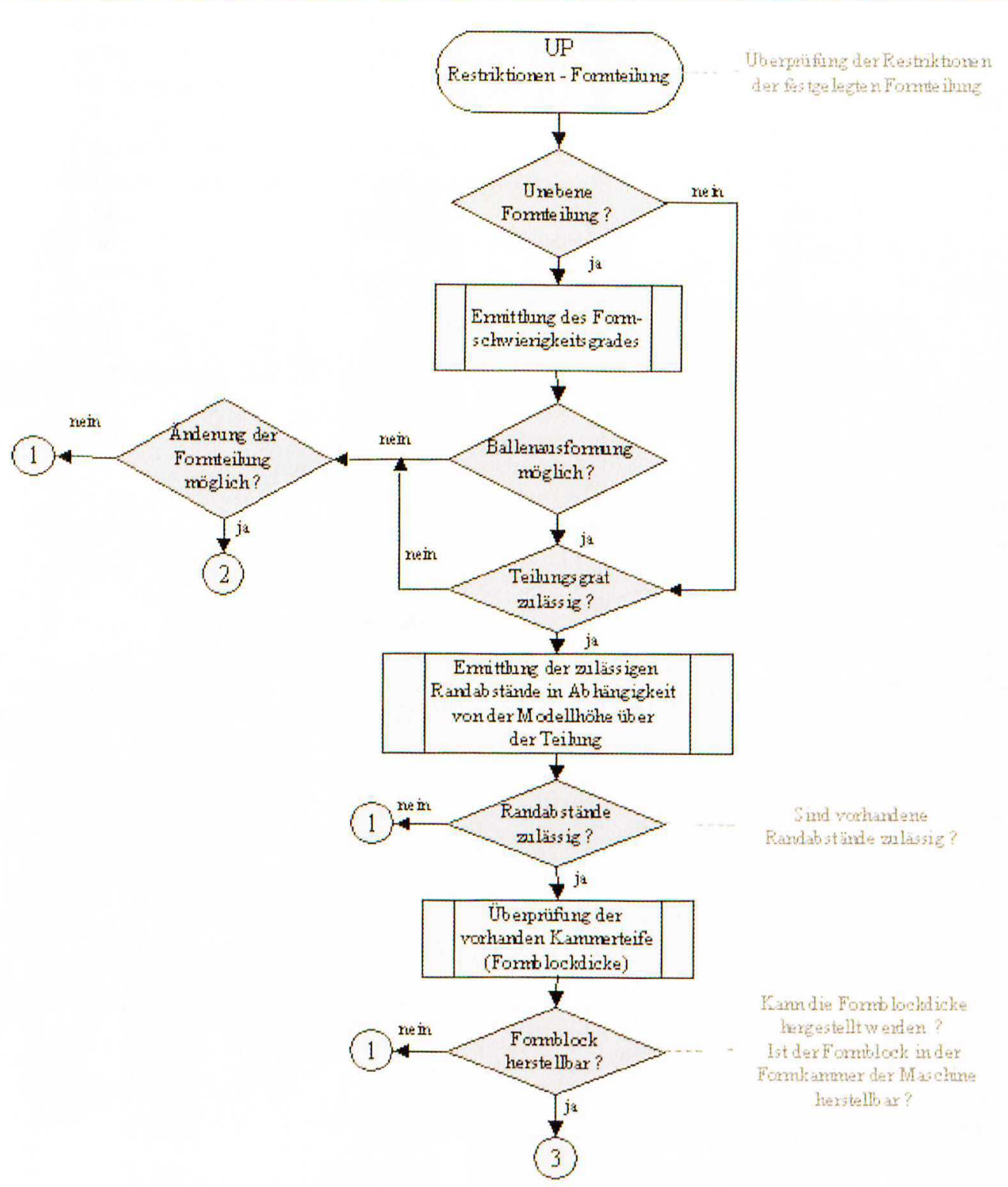


B Überprüfung des vorhandenen Verfahrensweges der Modellplatten mit dem maximal zulässigen Verfahrensweg.

C Korrektur des Einblasschlitzes um einen zulässigen Betrag, um den Modellverschleiß an beiden Modellplatten gleichmäßig gering zu halten.

\section{Kurzzeichenverzeichnis}

\begin{tabular}{|c|c|c|}
\hline Kurzzeichen & Erläuterung & Einheit \\
\hline A & $\begin{array}{l}\text { Dicke der Modellplatte auf der } \\
\text { Schwenkplattenseite der Maschine }\end{array}$ & $\mathrm{mm}$ \\
\hline B & $\begin{array}{l}\text { Dicke der Modellplatte auf der } \\
\text { Pressplattenseite der Maschine }\end{array}$ & $\mathrm{mm}$ \\
\hline $\mathrm{C}_{\mathrm{m}}$ & Gemessene Kammertiefe & $\mathrm{mm}$ \\
\hline $\mathrm{F}_{\mathrm{smax}}$ & Maximal zulässiger Formschwierigkeitsgrad & \\
\hline K & Verdichtbarkeit & $\mathrm{mm}$ \\
\hline $\mathrm{P}$ & $\begin{array}{l}\text { Modellhöhe auf der Schwenkplatte } \\
\text { (positives Modell) }\end{array}$ & $\mathrm{mm}$ \\
\hline $\mathrm{PP}$ & Pressplatte der Maschine & $\mathrm{mm}$ \\
\hline Q & $\begin{array}{l}\text { Modellhöhe auf der Pressplattenseite } \\
\text { (positives Modell) }\end{array}$ & $\mathrm{mm}$ \\
\hline $\mathrm{S}$ & Sicherheitsabstand & $\mathrm{mm}$ \\
\hline SP & Schwenkplatte der Maschine & \\
\hline $\mathrm{T}$ & Formblockdicke & $\mathrm{mm}$ \\
\hline$\underline{X}$ & Abstand der Heizplatte (SP) vom Einblasschlitz & $\mathrm{mm}$ \\
\hline Y & Abstand der Heizplatte (PP) vom Einblasschlitz & $\mathrm{mm}$ \\
\hline \multicolumn{3}{|l|}{ Restriktionen } \\
\hline $\mathrm{R}_{\text {Amin }}$ & $\begin{array}{l}\text { Restriktion für minimal zulässige } \\
\text { Schwenkplattendicke }\end{array}$ & $\mathrm{mm}$ \\
\hline$R_{B \min }$ & $\begin{array}{l}\text { Restriktion für minimal zulässige } \\
\text { Pressplattendicke }\end{array}$ & $\mathrm{mm}$ \\
\hline$R_{\text {Amax }}$ & $\begin{array}{l}\text { Restriktion für maximal zulässige } \\
\text { Schwenkplattendicke }\end{array}$ & $\mathrm{mm}$ \\
\hline$R_{B \max }$ & $\begin{array}{l}\text { Restriktion für maximal zulässige } \\
\text { Pressplattendicke }\end{array}$ & $\mathrm{mm}$ \\
\hline$R_{\text {Cmin }}$ & Minimal zulässige Kammertiefe & $\mathrm{mm}$ \\
\hline $\mathrm{R}_{\mathrm{Cmax}}$ & Maximal zulässige Kammertiefe & $\mathrm{mm}$ \\
\hline$R_{X \max }$ & Maximaler Einstellwert für X & $\mathrm{mm}$ \\
\hline$R_{Y_{\max }}$ & Maximaler Einstellwert für $Y$ & $\mathrm{~mm}$ \\
\hline$R_{(A+P)}$ & Maximale Modell- und Heizplattendicke (SP) & $\mathrm{mm}$ \\
\hline$R_{(B+Q)}$ & Maximale Modell- und Heizplattendicke (PP) & $\mathrm{mm}$ \\
\hline$R_{(P+Q)}$ & Maximale Modellhöhen (SP+PP) & $\mathrm{mm}$ \\
\hline
\end{tabular}

\section{A Vermeidung des Zusammenstoßens der Modellplatten nach dem Formprozess}

Um ein Zusammenstoßen der Modellplatten nach dem Verdichten des Formblockes zu vermeiden, wird zwischen den äußersten Modellkonturen ein Sicherheitsabstand von $\mathrm{S}=60 \mathrm{~mm}$ festgelegt.

\section{B Berechnung der Kammertiefe}

\section{Vergleich}

Jede DISAMATIC-Maschine hat eine zulässige Kammertiefe $C_{\text {zul }}$. Die vorhandene Kammertiefe $C_{\text {vorh }}$ muss berechnet werden. Ein Vergleich des errechneten Werts mit den Restriktionen $R_{\text {Cmin }}$ und $R_{\text {Cmax }}$ macht eine Aussage über die Realisierbarkeit der festgelegten Einformlage und Teilung am Gussteil.

\section{Modellhöhe}

Grundsätzlich kann eine Modellplatte aus positiven und/ oder negativen Modellausprägungen aufgebaut sein. Eine eindeutige Definition der einzelnen Parameter P und Q ist notwendig (siehe Abb. 16), um die Kammertiefe richtig berechnen zu können. Dabei gibt es Restriktionen $R_{\text {Amin }}, R_{\text {Amax }}, R_{B \text { min }}, R_{B \text { max }}, R_{(A+P)}, R_{(B+Q)}, R_{(P+Q)}$, die vor der Berechnung der Kammerteife überprüft werden müssen.

\section{Kompressibilität des Formsandes}

Die Kompressibilität des Formsandes ist die Differenz des Formballens vor und nach dem Preßvorgang der Maschine

$$
K=\frac{C_{m}-T}{C_{m}}
$$

Die Kompressibilität sollte in den Grenzen von 0.18 bis 0.25 liegen [6].

Berechnung der Kammertiefe

$$
C_{\text {vorh }}=A+B+\left[\frac{P+Q+S}{(1-K)}\right]
$$

\section{Korrektur des Einblasschlitzes}

Forderung für gleiche Abstände der Modellhöhen vom Einblasschlitz (siehe Abb. 16).
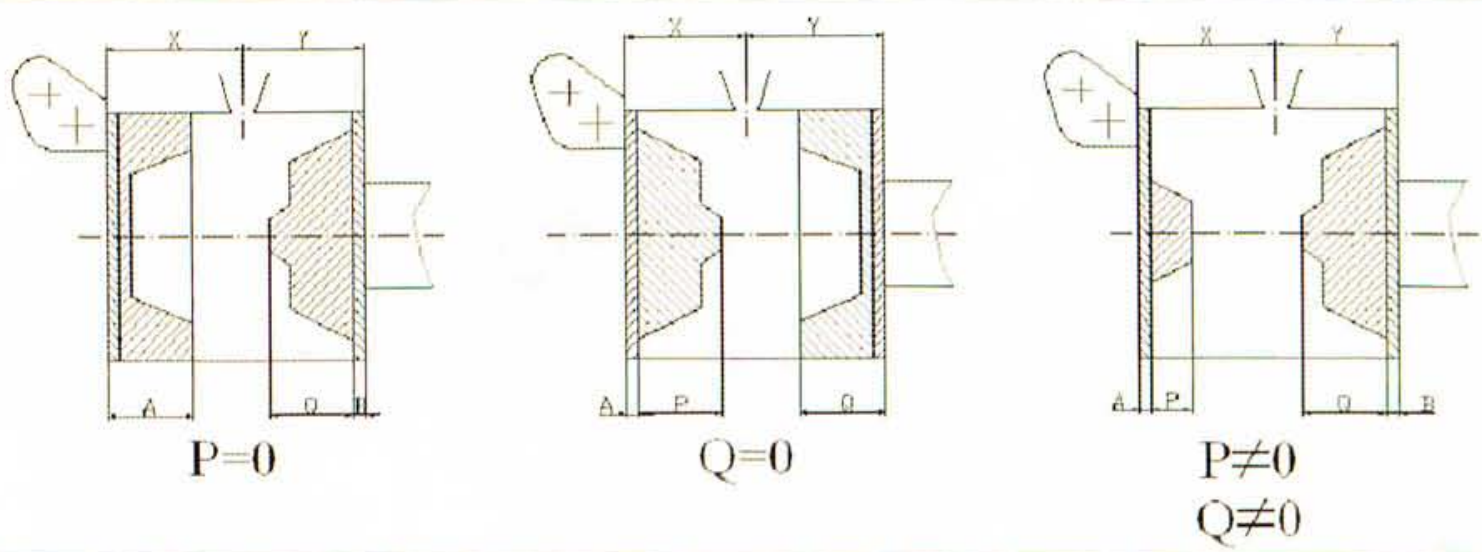

Abb. 16: Definition der Modellhöhen $P$ und $Q$ 


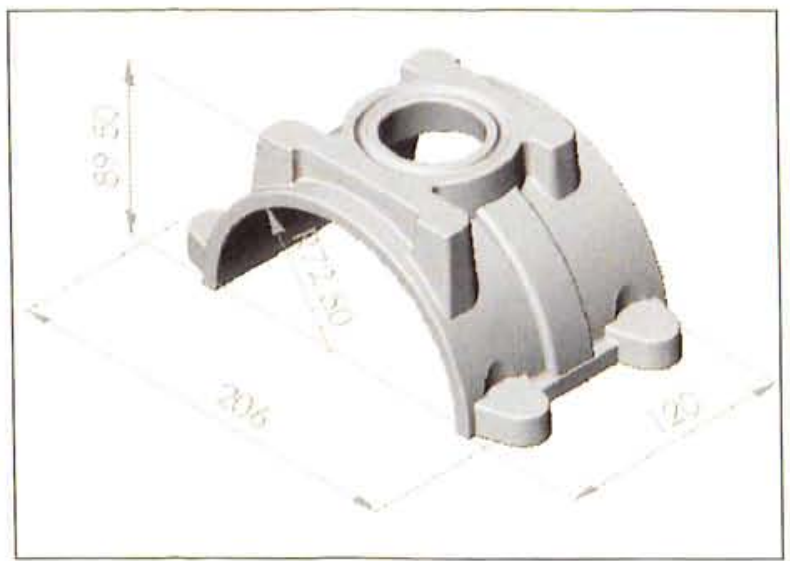

Abb. 17: Zu untersuchendes Gussteil auf der DISAMATIC 2110 MK3

$X-P-A=Y-Q-B$

Berechnung der Korrektur (siehe Abb. 16).

$Y=(Q+B-P-A)+X$

$X=Y-(Q+B-P-A)$

\subsection{Berechnungsbeispiel}

Das nach Abbildung 17 dargestellte Gussteil soll auf einer DISAMATIC 2110 MK3 Maschine geformt werden.

\section{Grobe Abschätzung der Realisierbarkeit}

Eine grobe Abschätzung der Realisierbarkeit kann durch einen Vergleich mit den angegebenen Formblockabmessungen in $|6|,|5|$ erfolgen. Um den Vergleich durchführen zu können, müssen die Einformlage und die Teilung festgelegt werden. Diese werden nach Abbildung 11 und Abbildung 14 festgelegt. Ein Formblock auf der DISAMATIC 2110 MK3 hat die Abmessungen (Höhe x Breite x Dicke) von $400 \times 500 \times(100-315)$ mm. Für die Plattenbelegung werden zwei Gussstiicke festgelegt. Nach Abbildung 14 ergibt sich eine gekrümmte Formteilung. Für diese Ballenausformung muss der Formschwierigkeitsgrad ïberprift werden $\mathrm{F}_{\text {smax }}=2$.

\section{Formblockbreite}

Die Breite der beiden Gussstiicke in der gewählten Einformlage ist $240 \mathrm{~mm}$. Die kleinste Wanddicke des Gussstücks beträgt $8 \mathrm{~mm}$. Die voraussichtliche Modellhöhe ohne Modellplatten über der Teilung beträgt $90 \mathrm{~mm}$. Die Randabstände nach $[6]$ sind dann seitlich $40 \mathrm{~mm}$. Berücksichtigung des Abstandes zwischen den beiden Gussstïcken mit $80 \mathrm{~mm}$. Die errechnete Formblockbreite ist $\left(240+2^{*} 40+80\right) \mathrm{mm}=400 \mathrm{~mm}$. Die zulässige Formblockbreite beträgt $500 \mathrm{~mm}$.

Ergebnis: Die Formblockbreite ist ausreichend.

\section{Formblockhöhe}

Die Höhe des Gussstiicks in der Einformlage ist $206 \mathrm{~mm}$. Die Randabstände nach $|6|$ sind oben $70 \mathrm{~mm}$ und unten $40 \mathrm{~mm}$. Die errechnete Formblockhöhe ist $(206+70+40) \mathrm{mm}=316 \mathrm{~mm}$. Die zulässige Formblockhöhe beträgt $400 \mathrm{~mm}$.

Ergebnis: Die Formblockhöhe ist ausreichend.

\section{Formblockdicke}

Die maximal mögliche Abmessung des Gussstücks in der Formblockdicke ist $89,5 \mathrm{~mm}$. Ein Sicherheitszuschlag von $60 \mathrm{~mm}$ ist notwenig, um eine Kollision der Modellplatten zu vermeiden. Der Formblock muß dann mindestens eine Dicke von $(89,52+60)$ mm aufweisen. Die errechnete Formblockdicke beträgt $239 \mathrm{~mm}$. Die zulässige Formblockdicke beträgt $315 \mathrm{~mm}$. Ergebnis: Die Formblockdicke ist ausreichend.

\section{Formschwierigkeitsgrad}

Die Ballenhöhe beträgt $72,5 \mathrm{~mm}$.

Die Ballenbreite beträgt $72,5 \mathrm{~mm}$ oder $120 \mathrm{~mm}$.

$\mathrm{F}_{\text {Svorh }}=1$ oder $\mathrm{F}_{\text {Svorh }}=0.6$

$\mathrm{F}_{\text {smax }}=2$

$\mathrm{F}_{\text {Svorh }}<\mathrm{F}_{\text {Smax }}$

Ergebnis: Der Formballen kann ausgeformt werden.

\section{Das Ergebnis der groben Überprüfung}

Nach einer groben Überprüfung unter Beachtung der Rohteilkriterien Einformlage und Teilung kann das Gussteil auf der Formanlage gefertigt werden.

\section{Genaue Überprüfung der Realisierbarkeit unter Einbeziehung weiterer Restriktionen}

Für eine genaue Überprïfung der Realisierbarkeit des in Abbildung 17 dargestellten Gussteils ist ein Entwurf der Formkammer zweckmäßig. Die in Abbildung 18 dargestellt Formkammer stellt bereits die Formkammer mit beiden fertig konfigurierten Modellplatten unter Einbeziehung aller Rohteilparameter dar. Für die aktuelle Aufgabenstellung wird nach dem Datenflussplan in Abbildung 10 vorgegangen. Das bedeutet, dass das Anschnittund Speisersystem für die nachfolgende Berechnung noch nicht berücksichtigt wird.

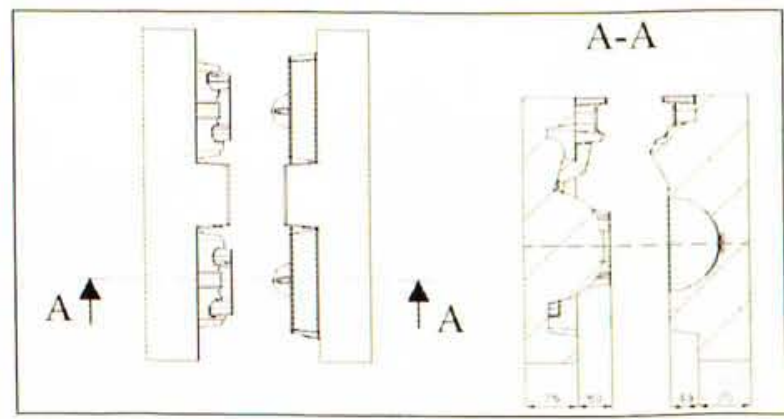

Abb. 18: Darstellung der Formkammer mit den Berechmungsparametern

Überprïfung der Restriktionen der verwendeten Modelle $A=75 \mathrm{~mm}$ liegt innerhalb vorgegebener Restriktionen. $\mathrm{R}_{\text {Amin }}=20 \mathrm{~mm}$

$R_{\text {Amax }}=120 \mathrm{~mm}$

$\mathrm{B}=75 \mathrm{~mm}$ liegt innerhalb vorgegebener Restriktionen.

$\mathrm{R}_{\mathrm{Bmin}}=20 \mathrm{~mm}$

$\mathrm{R}_{\mathrm{Bmax}}=150 \mathrm{~mm}$

$P=50 \mathrm{~mm}$

$\mathrm{Q}=40 \mathrm{~mm}$

$A+P=125 \mathrm{~mm}$ liegt innerhalb vorgegebener Restriktionen.

$R_{(A+P)}=185 \mathrm{~mm}$ 
$\mathrm{B}+\mathrm{Q}=115 \mathrm{~mm}$ liegt innerhalb vorgegebener

Restriktionen.

$\mathrm{R}_{(\mathrm{B}+\mathrm{Q})}=215 \mathrm{~mm}$

$\mathrm{P}+\mathrm{Q}=90 \mathrm{~mm}$ liegt innerhalb vorgegebener

Restriktionen.

$R_{(p+Q)}=240 \mathrm{~mm}$

Berechnung der Kammertiefe

$\mathrm{R}_{\text {Cmin }}=140 \mathrm{~mm}$

$R_{\mathrm{Cmax}}=440 \mathrm{~mm}$

Berechnung nach Formel 2 mit $\mathrm{K}=0.18$ und $\mathrm{S}=60$

$\mathrm{C}_{\text {vorh }}=332.9 \mathrm{~mm}$

Berechnung nach Formel 2 mit $\mathrm{K}=0.25$ und $\mathrm{S}=60$

Cvorh $=350 \mathrm{~mm}$

$\mathrm{C}_{\text {vorh }}<\mathrm{R}_{\text {Cmax }}$

$\mathrm{C}_{\text {vorh }}>\mathrm{R}_{\text {Cmin }}$

Die vorhandene Kammertiefe liegt innerhalb der vorgegebenen Restriktionen.

Korrektur der Position des Einblasschlitzes

$R_{\text {Xmax }}=60 \mathrm{~mm}$

$R_{\text {Ymax }}=150 \mathrm{~mm}$

Nach Gl. (4) ergibt sich für

$\mathrm{Y}=\mathrm{X}-10$

$\mathrm{X}=\mathrm{Y}+10$

Der Maschineneinstellparameter $\mathrm{Y}$ muss um $-10 \mathrm{~mm}$ oder der Maschineneinstellparameter $\mathrm{X}$ muss um +10 $\mathrm{mm}$ korrigiert werden. Beide Einstellungen liegen innerhalb des Verstellbereichs und sind möglich.

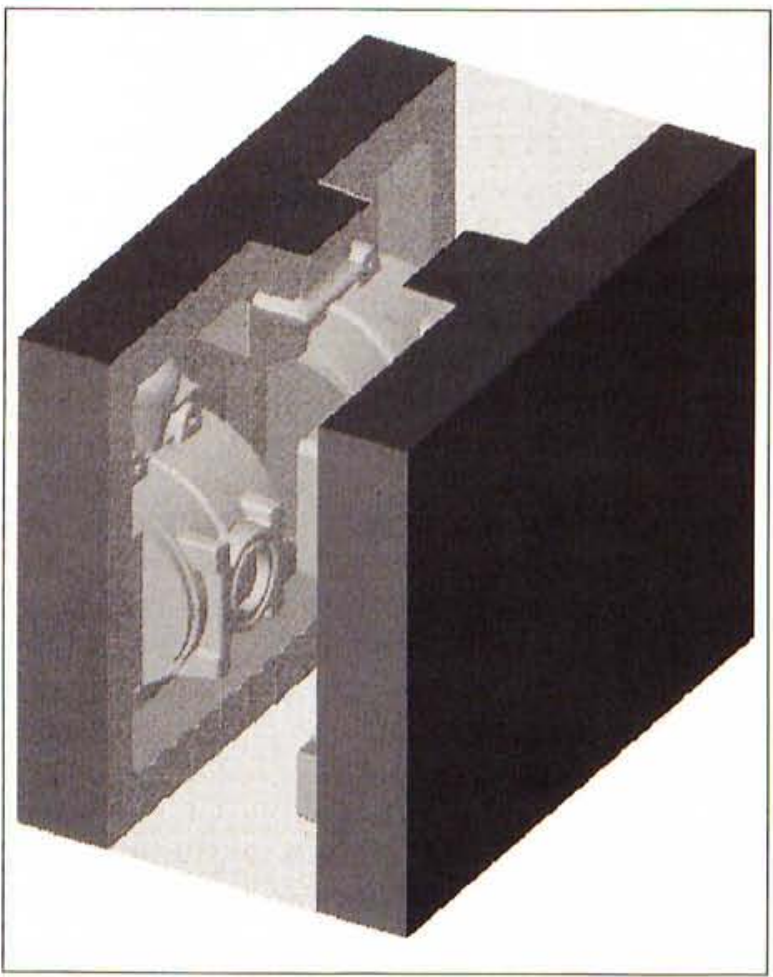

Abb. 19: Virtuelle Darstellung der Modellplattenpositionen zum Formblock nach der Verdichtung
Ergebnis:

Unter Berücksichtigung der maschinen- und werkzeugspezifischen Restriktionen bezogen auf eine gewählte Einformlage und Formteilung kann das in Abbildung 17 dargestellte Gussteil auf der Formanlage DISAMATIC 2110 MK3 geformt werden.

Mit Hilfe des 3D-CAD-Systems SolidWorks2000 können Gussteile, Modellplatten, Formblöcke und Formkammern übersichtlich dargestellt werden. Die 3D-Daten ermöglichen bei diesem Problem neben der Berechnung eine redundante sichere Restriktionskontrolle. Bei erstellten Geometriedaten des Gussteils in der Arbeitsvorbereitung können der Modellbau (CAM) und die Qualitätssicherung (CAQ) die CAD-Datenbasis im Sinne einer lïckenlosen, änderungsfreundlichen und produktiven Prozesskette nutzen. Abbildung 19 stellt die simulierte Formkammer nach dem Pressvorgang dar. Durch das verwendete Volumenmodell kann auch der Einsatz von Formsand und Kreislaufmaterial mit dem CAD-System berechnet werden und gibt Aufschluss für weitere wirtschaftliche Analysen.

\section{Zusammenfassung und Ausblick}

Die Betrachtung von produktiven Werkzeugmaschinen für das Formgießen in verlorenen Formen gewinnt mit der zunehmenden Anwendung von Rapid Prototyping Verfahren und der industriellen Forderung der metallverarbeitenden Industrie nach mehr Gusswerkstüicken mit den Vorzügen einer gestalterischen Freiheit an Bedeutung. Dabei werden produktive kostengünstige Formverfahren favorisiert. In diesem Artikel wurde eine produktive kastenlose Disamatic-Formanlage mit vertikaler Formteilung vorgestellt. Ausgehend von den technischen Details der Maschine bis hin zur Berïcksichtigung des Einsatzes in der Fertigungsplanung durch Überpruifung von Restriktionen, wird ein grundlegender Überblick über die Problemstellungen beim Einsatz der Formanlage in einer Kundengießerei gegeben.

In diesem Artikel wurde nur die Berechnung der Restriktionen bezüglich einer festgelegten Einformlage und Formteilung vorgestellt. Weitere zu lösende Probleme stellt der Ablauf von Abbildung 10 dar. In den abzuarbeitenden Unterprogrammen zur Generierung des Rohteils und zur Herstellung des Urformwerkzeugs ist es notwendig, weitere maschinenspezifische Parameter, wie z. B. der Einsatz des Kerneinlegers oder die Dimensionierung des Anschnitt- und Speisersystems für eine Realisierbarkeit in der Auftragsfertigung zu überprüfen.

\section{Danksagung}

Der Autor dankt an dieser Stelle der Firma „DuktilGuss-Fürstenwalde GmbH“ für die kooperative und herzliche Zusammenarbeit. Aufgrund der dort durchgeführten Untersuchungen war es möglich, den Artikel, vor allem im letzten Teil, mit praxisbezogenen Beispielen zu illustrieren. 


\section{Literatur}

[1] Ambos, E.; Hartmann, R.; Lichtenberg, H.: Fertigungsgerechtes Gestalten von Gußstïcken, Vieweg, Wiesbaden 1992

[2] Ambos, E.; Hofmann, 1.; Scheler, R.; Krötzsch, S.; Pfisterer, W.; Miersch, N.: Effektivitätssteigerung durch Einsatz rechentechnischer Lösungen in der Arbeitsvorbereitung, Giesserei 87 (2000) 19, S. 39-45

[3] Brunhuber, E.: Gießerei-Lexikon, Schiele \& Schön, 17. Auflage, 1997

[4] DGV - Deutscher Gießereiverband: Wer gießt was? Firmen, Produkte, Vefahren. Die Eisen-, Stahl- und Tempergießereien im Deutschen Gießereiverband, 5. Auflage

[5] Georg Fischer Disa GmbH: DISAMATIC 2110 MK3. Eine kluge Wahl. Prospekt, November 1996

[6] Georg Fischer Disa GmbH: Handbuch der Anwendungstechnik für Disamatic 2110 MK2 Sandformsystem. September 1995

[7] Georg Fischer Disa GmbH: List of Georg Fischer disa AVS Equipment, sold as per March 31, 1999

[8] Hasse, S.: Guß- und Gefügefehler. Erkennung, Deutung und Vermeidung von Guß- und Gefügefehlern bei der Erzeugung von gegossenen Komponenten, Schiele \& Schön, Berlin 1999

[9] Hofmann, 1.; Spangenberg, J.; Miersch, N.; Ambos, E.; Pfisterer, W.: Rechnergestïtzte Generierung des Rohteiles im Rahmen der Angebotsbearbeitung fuir Neuteile - Optimierungsstrategien für Gußstücke. Tagungsband zum Eröffnungskolloquium „Innovative Gießerei“. Teilprojekt 1: Indirekte Wertschöpfungskette FSP 2: Rationellere Angebotsbearbeitung, Otto-von-Guericke-Universität Magdeburg, 17. April 1998

[10] Müller, G.: Lexikon Technologie. Metallverarbeitende Industrie. Verlag Europa Lehrmittel, 2. Auflage, Haan 1992

[11] Roll, F:: Handbuch der Gießerei-Technik. Zweiter Band/1. Teil. Konstruktion, Anschnitt-Technik, Allgemeine Formverfahren, Spezielle Formsande. Springer Verlag, Berlin/ Heidelberg 1970

[12] Weck, M.: Werkzeugmaschinen Fertigungssysteme 1. Maschinenarten und Anwendungsbereiche Springer Verlag, 5. Auflage, Berlin/Heidelberg/New York 1998

\section{Autor}

\section{Dipl.-Ing. Norbert Miersch}

Technische Fachhochschule Wildau Fachbereich Ingenieurwesen/Wirtschaftsingenieurwesen Tel. (0 33 75) 508-161

E-Mail: miersch@hrz.tfh-wildau.de 